\title{
A Novel Robotic Laser Ablation System for Precision Neurosurgery with Intraoperative 5-ALA-Induced PpIX Fluorescence Detection
}

\author{
Masafumi Noguchi ${ }^{1}$, Eisuke Aoki ${ }^{1}$, Daiki Yoshida, Etsuko Kobayashi ${ }^{1}$, \\ Shigeru Omori ${ }^{2}$, Yoshihiro Muragaki ${ }^{3}$, Hiroshi Iseki ${ }^{3}$, Katsushige Nakamura ${ }^{4}$, \\ and Ichiro Sakuma ${ }^{1}$ \\ ${ }^{1}$ Graduate School of Frontier Sciences, The University of Tokyo, \\ 7-3-1, Hongo, Bunkyo-ku, Tokyo 113-8656, Japan \\ \{masamasa, aoki, dai, etsuko\}@miki.pe.u-tokyo.ac.jp, \\ isakuma@k.u-tokyo.ac.jp \\ ${ }^{2}$ R\&D Center, Terumo Corporation, 1500 Inokuchi, Nakai-machi, \\ Ashigarakami-gun, Kanagawa 259-0151, Japan \\ Shigeru-Oomori@terumo.co.jp \\ ${ }^{3}$ Faculty of Advanced Techno-surgery, Institute of Biomedical Engineering \\ and Science, Graduate School of Medicine, Tokyo Women's Medical University, \\ 8-1 Kawada-cho, Shinjuku-ku, Tokyo 162-8666, Japan \\ \{ymuragaki, hiseki\} aabmes. twmu.ac.jp \\ ${ }^{4}$ Mitaka Ko-Ki Corporation, 5-1-4 Osawa, Mitaka-shi, Tokyo 181-0051, Japan
}

\begin{abstract}
We developed a combined system of tumor detection by 5-ALAinduced PpIX fluorescence and precise ablation by micro laser for the first time, with an automatic focusing and robotic scanning mechanism for the brain surface. 5-ALA accumulates on tumors to be metabolized to become PpIX that is a fluorescent. Intra-operative detection of 5-ALA induced PpIX fluorescence provides useful information for tumor detection. The wavelength of the micro laser is $2.8 \mu \mathrm{m}$ close to the absorption band of water. This laser is effective only on the surface of brain tissue, enabling precise ablation at the boundary between tumor and normal tissue identified by intra-operative 5-ALA induced fluorescence. Combination tests of the fluorescence measurement and the laser ablation were performed, and it was possible to extract the area with fluorescence appropriately from the measurement data, and the micro laser with automatically scanning selectively ablated the extracted area.
\end{abstract}

\section{Introduction}

In current neurosurgical practice, surgeons can remove most of a tumor with an accuracy of a few millimeters by using a combination of conventional surgical instruments, such as an electric cautery, and a computer-aided navigation system based on diagnostic images, such as MR and CT images. Residual tumor, especially if the malignant tumor like glioma, may impair the prognosis of the patients and it is necessary to remove as much of the tumor as possible while keeping the normal tissue 
intact. However, it is difficult to know the exact boundary between tumor and normal tissue, and excessive ablation of the normal brain tissue will damage its function.

In craniotomy procedure, deformation of the brain tissue, called "brain shift", occurs due to cerebrospinal fluid leakage and surgical interventions. In some cases, brain shift reaches to several tens of millimeters and continuously increases during the procedure [1]. This requires navigation based on intraoperative MR images. This navigation, however, contains a few millimeters of errors at a maximum caused by a registration of preoperative diagnosis images [1] and intraoperatiove images and an accuracy of the position tracking using such as an optical marker. Furthermore, there is a tradeoff between high frequency of image acquisition for more accurate navigation and not time-consuming imaging.

Similarly, the accuracy of conventional surgical procedures is a few millimeters for removal of residual tumors. Therefore, we desired to achieve a more precise operation with an accuracy of sub-millimeters. To that end, each of a measurement and removal of residual tumors has to realize this accuracy.

To solve these problems, we have proposed a novel approach to therapy using 5aminolevulinic acid (5-ALA) [2][3] and a micro-laser ablation system [4], with the boundary between the tumor and the normal tissue distinguished by the 5-ALAinduced protoporphyrin IX (PpIX) fluorescence in the tumor and with accurate ablation of the tumor with the micro laser. 5-ALA, which is orally administrated to a patient, accumulates on tumors to be metabolized to become PpIX that is a fluorescent substance [5]. The wavelength of the micro laser is $2.8 \mu \mathrm{m}$. Light with this wavelength is mostly absorbed by water, and therefore this laser is effective only on the surface of brain tissue, enabling precise ablation at the boundary between tumor and normal tissue [4].

In this paper, we developed a combined system of tumor detection using 5-ALA and precise ablation by micro laser, with an automatic focusing (AF) and robotic scanning mechanism for the brain surface. This system is designed for possible localized pinpoint detection of the tumor, then ablating the fluorescent area with stepping driven precise position control in the whole system. This is first attempt to integrate intraoperative fluorescence detection and high precision laser ablation system. In addition, each of the measurement and ablation is performed under the robotic position controlling.

\section{Materials and Methods}

In this chapter, the tumor detection using 5-ALA-induced PpIX fluorescence, the micro laser module, the automatic focusing and robotic scanning system, and the whole system integration are described. Finally we proposed the experimental procedure for combining these surgical processes.

\subsection{Intraoperative Detection System for Brain Tumor Using 5-ALA}

Fluorescence of PpIX is fully observed a few hours after orally administrated 5-ALA. Irradiating near-ultraviolet light of around $400 \mathrm{~nm}$, PpIX emits the fluorescence of the wavelength of $635 \mathrm{~nm}$ at peak intensity from the brain tissue. 
Applying a highly sensitive camera is one of the reasonable ways for intraoperative detection of PpIX fluorescence. Although it is possible to acquire a wide-area 2dimensional data at one time, obtained data contains only light intensity and simple color information. As PpIX fluorescence is so much weaker than the excitation light and a guide laser of the AF system, it requires the optical narrow-band-pass filter to cut off the other light sources. Nevertheless, it is difficult to separate the fluorescent component from the intensity and the color information of the image signal. Therefore, we chose the use of a spectral photometer, easily extracting the peak intensity of the fluorescence. The spectral data contains important information about tumors; for example, a spectral shape and a peak wavelength possibly vary with a density of tumor cells, a class of tumors, and other conditions of tissues. In the future task, we consider acquisitions of the functional information of tumors by spectral analysis.

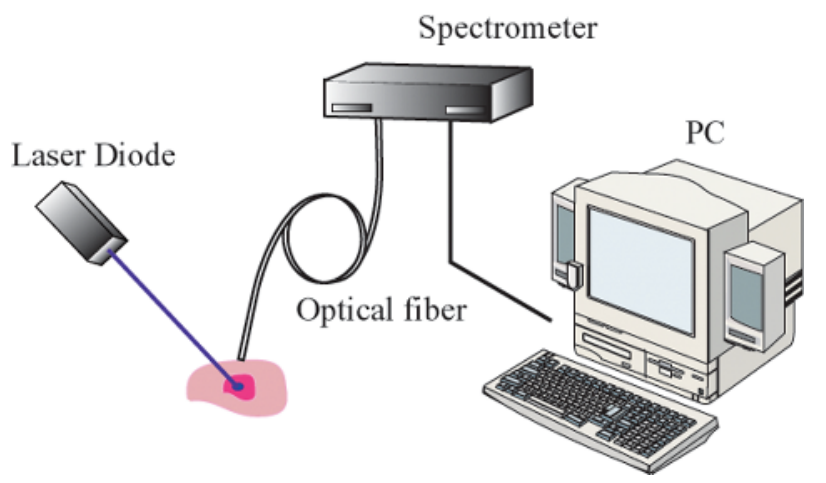

Fig. 1. Tumor detection system using 5-ALA-induces PpIX fluorescence
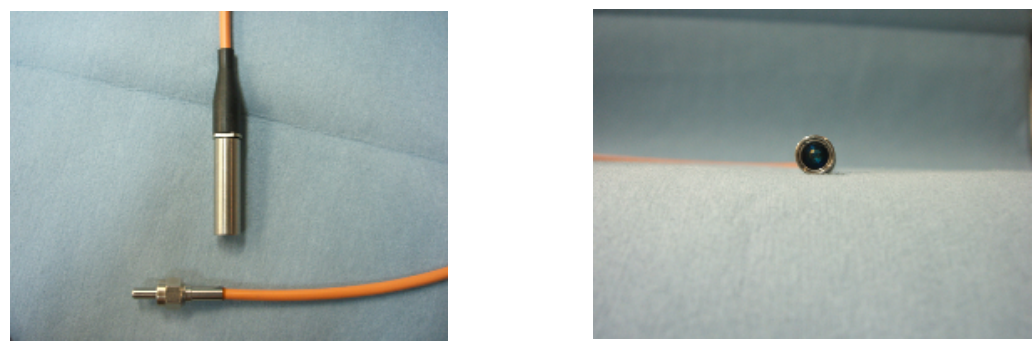

Fig. 2. Fluorescence detection probe

PpIX fluorescence was collected by a detection probe and guided into a spectrophotometer through an optical single-mode fiber, then performed spectral analysis (Fig. 1). The detector has a diameter of $8 \mathrm{~mm}$, using aspheric lenses to correct aberrations. The detection resolution is set to $0.6 \mathrm{~mm}$, considering a tradeoff between not decreasing of the acquired light intensity in proportion to the square of the resolution diameter and improving the accuracy of the measurement (Fig.2). A band-pass filter, which transmits over $60 \%$ at $635 \mathrm{~nm}$ and up to $5 \%$ at $670 \mathrm{~nm}$, was 
fixed on the tip of the detector, cutting off the excitation light and the guide laser (peak at $670 \mathrm{~nm}$ ) of the AF system. The working distance of the detector is $16 \mathrm{~mm}$ to a tolerance of plus or minus $0.5 \mathrm{~mm}$.

\subsection{Micro Ablation Laser Module}

For ablation of tumor tissues, we used a mid-infrared continuous-wave laser with a wavelength of $2.8 \mu \mathrm{m}$, being output by a microchip solid-state laser on the tip of a laser probe [4]. The pumping light source for the solid-state laser is a near-infrared diode laser with a wavelength of $970 \mathrm{~nm}$, guided through a quartz optical fiber to the laser probe.

As the light wavelength around $3 \mu \mathrm{m}$ has strong absorption feature by water, this laser is effective only on the surface of the living tissue, and it can make a precise ablation with a low output of $0.2 \mathrm{~W}$ or less. The laser beam is focused to a diameter of 0.1 to $0.15 \mathrm{~mm}$ with a lens, and an ablation groove is formed equivalent to the spot diameter in the soft vital tissue. The working distance of the laser probe is $15 \mathrm{~mm} \pm 1 \mathrm{~mm}$.

\subsection{Automatic Focusing and Robotic Scanning System}

Both the fluorescence detection probe and the micro laser probe have each working distance, and this requires an $\mathrm{AF}$ mechanism, constantly maintaining the distance from the brain surface. In this practice, we used an AF system designed based on the three-dimensional measurement system (Mitaka Ko-ki Co., Ltd.) (Fig. 3). In this system, position measurement was performed using a confocal optical mechanism and the guide laser was picked up with a split photodiode, enabling a focusing with an accuracy of micrometers. The wavelength of the guide laser is $670 \mathrm{~nm}$. This system was coupled with 2-axial automatic stepping drive stage and can make a robotic scanning on the surface of the brain.

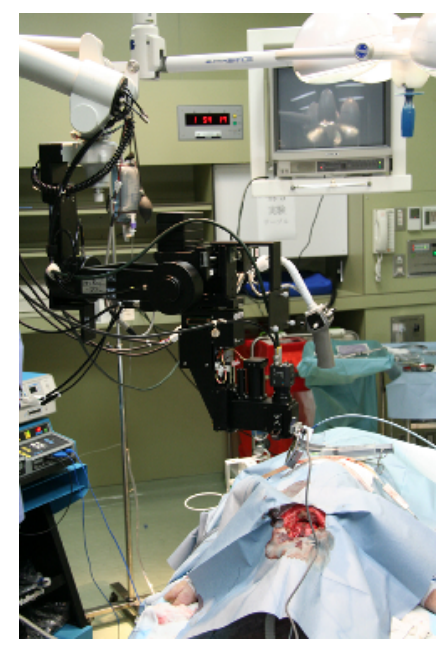

Fig. 3. Automatic focusing and robotic scanning system 


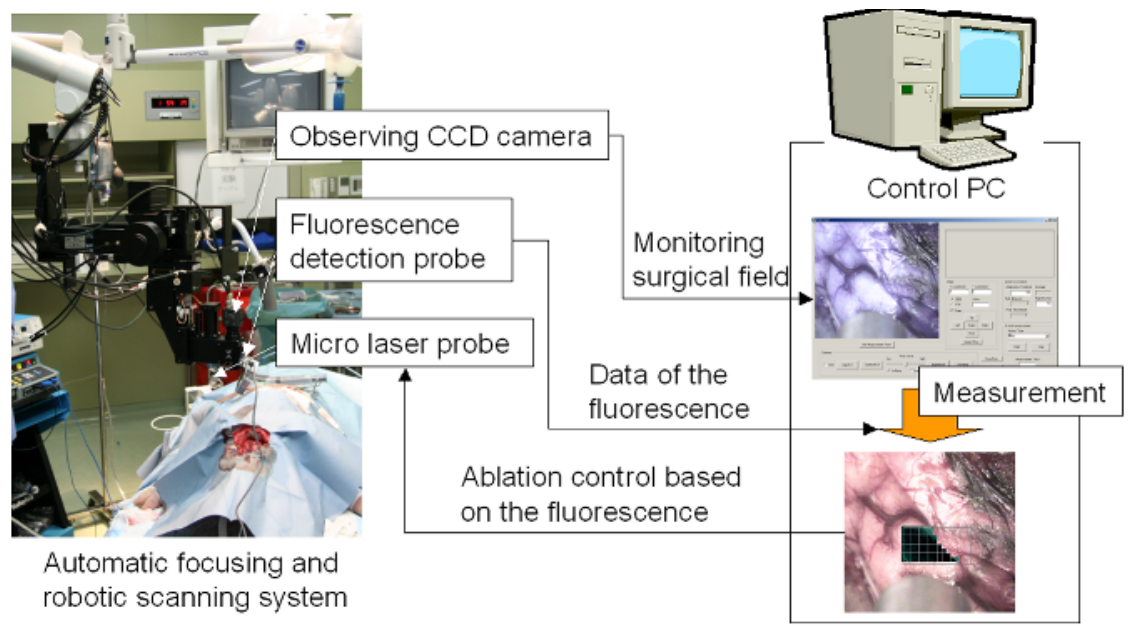

Fig. 4. System configuration

\subsection{System Integration}

Fig. 4 shows the system configuration in this paper. The fluorescence detection probe and the micro laser probe were attached to the AF system. The data from the spectral photometer was stored into a personal computer (PC). Switching of the micro laser and scanning with the stepping drive were both controlled by the PC. We can observe surgical field view by a CCD camera in the AF system. This image was used to control electric motors to position the fluorescence measurement system and the laser ablation system.

\subsection{Experimental Procedure}

\section{Measurement of Fluorescence Signal}

Measurement area was defined on a CCD camera view of the AF system and sectioned in a grid pattern. Measurement was performed on each grip point with raster
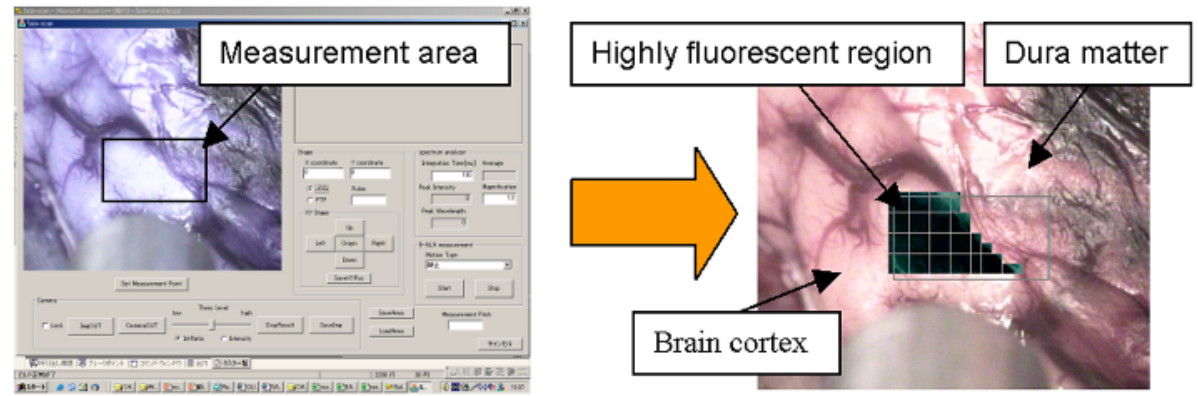

Fig. 5. An example of the measurement procedure of the fluorescence on a porcine brain 
scanning. A point in the measurement area was regarded to have same fluorescent property with nearest grid point. A grid interval was determined to $0.4 \mathrm{~mm}$, considering that the grid square was included in the circle of the measurement resolution of $0.6 \mathrm{~mm}$. As a measurement required more than a few hundred of milliseconds, it took several tens of seconds or more to measure a few millimeters square. Fig. 5 shows an example of the fluorescence measurement procedure.

\section{Ablation of the Target Area}

A threshold was set for the intensity level of measured fluorescence. In this paper, a surgeon or an experimenter determined the threshold, viewing the measurement area on the CCD camera image. Then scanning was started, and the micro laser was automatically irradiated on the region over the threshold.

\section{Results}

Combination tests of the tumor detection system and the micro ablation laser module were performed for a biomedical simulant material (phantom) and a porcine brain.

\subsection{Phantom Experiment}

The phantom was composed of agar plate containing Intralipid-10\% that is intraenous lipid emulsion and used for scattering medium [6]. The concentration of Intralipid-10\% was adjusted so that the scattering coefficient became $3 \mathrm{~cm}^{-1}$, which is similar to that of Glioma [6][7]. A half part of the phantom contained PpIX to emit fluorescence and the other half part did not contain PpIX.

Fig. 6(a) shows one of the results of experiments. Black rectangular area stands for the scanned area by the system. The system could identify the boundary between the area with fluorescence and without fluorescence, and could precisely ablate the fluorescent area with automatically scanning.

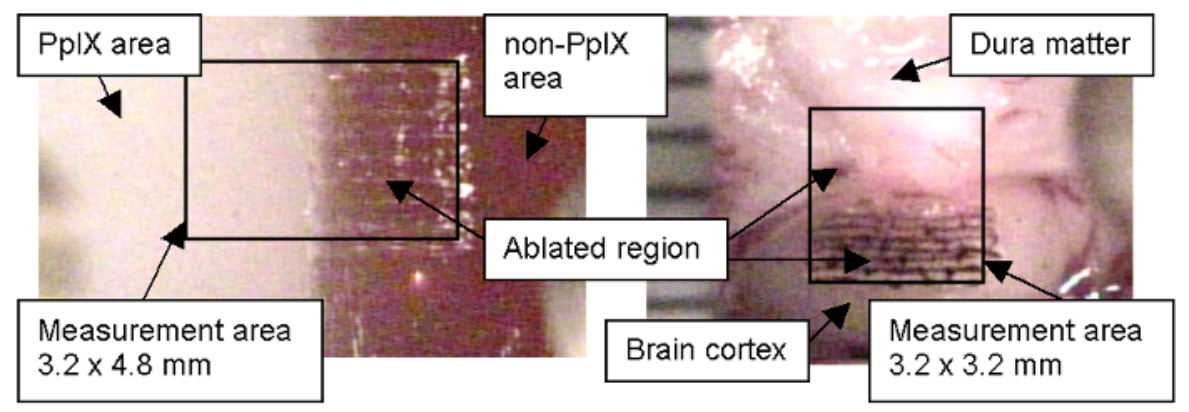

Fig. 6. Result of the combination tests of the tumor detection system and the micro ablation laser module 


\subsection{Porcine Brain Experiment}

The target was the surface of a porcine brain exposed by craniotomy under anesthesia. Before the experiment, 5-ALA was administrated in sufficient quantities to accumulate on a normal brain tissue and metabolize to become PpIX. A half of the measurement area was covered with dura matter, where the fluorescence was not observed, and the other half was exposing brain cortex.

Fig. 6(b) shows one of the results of experiments. Black rectangular area stands for the scanned area by the system. The dura matter covering the porcine brain could not completely block the fluorescence. We could identify weak signal of fluorescence from some part of the dura matter. As shown in Fig. 6(b), ablation laser was irradiated to such area on the covered region. We could successfully identify and ablate the fluorescent area of the porcine brain based on the fluorescence data. The AF system functioned properly even for porcine brain and was effective to stabilize both of the conditions of the measurement and the laser ablation.

\section{Discussion and Conclusions}

We developed a combined system of tumor detection by 5-ALA-induced PpIX fluorescence and precise ablation by micro laser for the first time, with an automatic focusing and robotic scanning mechanism for the brain surface. In this system, ablation was performed based on the fluorescent information under the robotic position controlling.

Combination tests of the fluorescence measurement and the laser ablation were performed for a biomedical simulant material (phantom) and a porcine brain. Measurement areas of the phantom and the porcine brain were both separated into fluorescent part and non-fluorescent part. In each test, it was possible to extract the area with fluorescence appropriately from the measurement data, and the extracted area was selectively ablated by the micro laser with automatically scanning.

In this practice, the experimental targets were clearly separated into the area with fluorescence and without fluorescence. In clinical cases, the boundaries between tumors and normal tissues are often unclear, and tumors invade normal tissues. Therefore, thresholding of a fluorescence data and an extraction of the area for ablation will have problems, and are considered making some automation process. Solutions for these problems are desired in the future work. To automatically discriminate the tumor region by fluorescence data, a multiple classification analysis based on another spectral features not only peak intensity of the fluorescence will be investigated.

\section{Acknowledgments}

We wish to thank many people and groups who have contributed to this effort. Research on medical devices for analyzing, supporting and substituting the function of human body funded by Ministry of Health, Labour and Welfare. 


\section{References}

1. Clatz Oliver, et. al, Robust nonrigid registration to capture brain shift from intraoperative MRI, IEEE Transactions on Medical Imaging, vol. 24, no. 11, November, pp. 1417-1427, 2005.

2. T. Maruyama, et al, "Intraoperative detection of malignant gliomas using 5-Aminolevulinic acid induced protoporphyrin fluorescence, openMRI and real-time navigation system," Computer Assisted Radiology and Surgery, vol. 15, pp. 270-275, 2001.

3. K. Shimizu, et al, "Application of blue semiconductor laser to measurement of 5-ALA induced fluorescence for intraoperative detection of brain tumor," Proceedings of 6th Japan-France Congress on Mechatronics and 4th Asia-Europe Congress on Mechatronics, 2003, pp. 135-140.

4. S. Omori, Y. Muragaki, I. Sakuma, and H. Iseki, "Robotic laser surgery with $\lambda=2.8 \mathrm{um}$ microlaser in neurosurgery," Journal of Robotics and Mechatronics, vol. 16, no. 2, pp. 122-128, 2004.

5. M. Norio, et al, In light of recent developments, application of fluorescence spectral analysis in tumor diagnosis, Applied Spectroscopy Reviews, vol. 39, no. 4, November, pp. 437-455, 2004.

6. J. van Staveren, J. M. Moes, J. van Marle, A. Prahl, J. C. van Germert, "Light scattering in Intralipid- $10 \%$ in the wavelength range of 400-1100 nm," Appl Opt, vol. 30, pp. 45074514, 1991.

7. Sterenborg HJ, et al, "The spectral dependence of the optical properties of the human brain,” Lasers Med Sci, vol. 4, pp. 221-227, 1989. 